\title{
Antagonists and partial agonists
}

A FAVOURITE technique for studying T-cell specificity has been to offer a set of T-cell clones specific for a particular antigenic peptide a set of synthetic peptides that differ in one or more positions. Such experiments have shown that amino acids at some positions in the peptide are important for binding to the MHC molecule while others affect only recognition by the $T$ cell, and are thought to contact the T-cell antigen receptor. Conservative substitutions in most of the residues not essential for MHC binding are still recognized by most T-cell clones, but higher concentrations of the cross-reactive peptide analogue are frequently required to elicit an equivalent response; presum. ably, the analogues have a lower affinity for the T-cell antigen receptor, and so a higher concentration of peptide/MHC molecules on the antigen-presenting cell is required to give an equivalent level of antigen receptor occupancy.

This picture has been complicated by the finding that some peptide analogues have antigen-specific effects on $T$ cells that are different in kind from those of the antigenic peptide. For example, some analogues act as specific competitive antagonists for a T-cell clone: they block all measurable $\mathrm{T}$-cell responses to an antigenic peptide when present in excess over the antigenic peptide ${ }^{5}$. And other analogues cannot induce interleukin-2 production and T-cell proliferation at any concentration, but can still elicit other responses from a T-cell clone, such as cellular cytotoxicity or T-cell help for B-cell responses ${ }^{6}$. Some of these peptides with partial agonist properties are also antagonists when presented together with an antigenic peptide ${ }^{7}$.

The discovery of partial agonist and antagonist peptides suggests that the $T$ cell has some way to measure the quality of the interaction of an individual antigen receptor with an MHC/peptide complex, as well as overall receptor occupancy. Perhaps the half-life and precise alignment of the individual TCR/peptide/MHC complex determine the kind of signal by limiting the assembly of a signalling complex (for example, MHC dimeriza. tion or CD4 association).

Antagonism could provide a safe solu. tion to the paradox of positive and negative selection in the thymus. The hypothesis is that a self peptide that positively selects a $\mathrm{T}$ cell in the thymus would interact with the T-cell receptor in the same range of affinity or in the same way as an antagonist peptide for a mature $T$ cell. In this way, positive and negative selection could occur on largely nonoverlapping sets of self peptides. More importantly, autoimmunity would be neatly avoided were a mature $T$ cell to encounter its selecting self peptide on some vulnerable tissue in the body at higher concentrations than in the thymus, because the self peptide would antagonize rather than stimulate the $T$ cell.

Superficially, the results of Bevan and colleagues $^{1}$ support this view, because they found that the peptides that positively select a particular TCR are a sub. set of the peptides previously found to be antagonists for that TCR in a mature T cell. But the correlation may be more ap. parent than real, and reflect the levels of representation of the tested peptides on the thymus epithelium. If higher levels of representation could be achieved, it is possible that more distantly related peptides might positively select, as reported by Tonegawa and co-workers ${ }^{2}$. Moreover, both groups found that the same peptide can act in both positive and negative selection for a particular T-cell receptor, depending on peptide/MHC concentration on the surface of the selecting cell.

P.M. \& D.C.P strongly antigenic for the TCR in a mature T cell line. Pamela Ohashi's group, using the same LCMV TCR but the type of knockout mouse used by Bevan and colleagues, has also found that the antigenic peptide can positively select its TCR (ref. 3). The native ovalbumin peptide, on the other hand, did not select its TCR, even when it was added at very low concentrations to the thymus organ cultures. So there may be an intrinsic affinity threshold above which only negative selection is possible. Also, in the ovalbumin peptide studies, some antagonist peptides could positively select whereas others, which appeared to be equally good antagonists, could not. It is possible that mature $\mathrm{T}$ cells and thymocytes can discriminate between ligands other than by simple receptor occupancy (see box). Another study provides evidence that an antagonist peptide may antagonize positive selection in fetal thymic organ culture $^{4}$, a possibility inconsistent with any simple receptor occupancy model. Perhaps a hetter understanding of the mechanisms of antagonism will yet shed light on positive selection.

Although the new data show clearly that positive selection is peptide specific and dependent on TCR affinity and ligand concentration, the degree of specificity or degeneracy in positive selection in a normal thymus remains to be determined. For example, Tonegawa's group has re- ported that a combination of two particular exogenously added peptides selected as many heterogeneous $\mathrm{T}$ cells (in nonTCR transgenic mice) as a mixture of the many class I-binding peptides isolated from spleen cells, a result which is difficult to reconcile with the peptide specificity of positive selection demonstrated in the current papers. Thus, the matter of which and how many peptide/MHC combinations contribute to positive selection of a particular TCR in a normal thymus remains open.

There are other outstanding questions, the most important of which concerns the ability of developing $T$ cells to detect very low affinity, low ligand densities with their TCRs. Immature thymocytes bear 5-10 times fewer TCRs per cell than mature $\mathrm{T}$ cells, yet they can detect ligands and ligand concentrations which are apparently not seen by mature $\mathrm{T}$ cells. Given that the affinities of TCRs for their antigenic peptide/MHC ligands are very low, some $10^{-5} \mathrm{M}$ according to the few published measurements, how do thymocytes discriminate between ligands with affinities much less than this? Is the exquisite sensitivity of immature thymocytes aided by the fact that their TCR/CD3 complex is already partially phosphorylated or that their TCRs are incompletely engaged by CD3? Do immature thymocytes possess as yet undiscovered ligands that could stabilize very weak interactions between TCR and peptide/MHC? What are the signals for positive selection that require thymic organ culture and cannot yet be reproduced in cell suspensions? Finally, are the rules for positive selection of thymocytes destined to be restricted by class II MHC proteins similar to those for class I?

Nonetheless, our guess is that the problem of positive selection is cracking before our eyes. The phenomenon involves an interaction between the receptors of developing thymocytes and peptide/MHC complexes at affinities or ligand densities too low to drive T-cell activation or thymocyte death. This is truly a case of a little of what you fancy does you good.

Philippa Marrack is in the Howard Hughes Medical Institute and the Department of Medicine at the National Jewish Center for Immunology and Respiratory Diseases, Denver, Colorado 80303, USA. David C. Parker is in the Department of Molecular Genetics and Microbiology, University of Massachusetts Medical School, Worcester, Massachusetts 01655, USA.

1. Hogquist. K. A. etal. Cell 76, 17-27 (1994)

2. Ashton-Rickardt, P. G. et al. Cell 76, 651-663(1994)

3. Sebzda, E. etai. Science 263, 1615-1618 (1994).

4. Spain, L. M., Jorgenson, J. L., Davis, M. M. \& Berg, L. J. J. Immun. 152, 1709-1717 (1994).

5. De Magistris, M. T. et al. Cell68, 625-634 (1992).

6. Evavold, B. D., Sloan-Lancaster, J.\&Allen, P. M. Immun Today 14, 602-609 (1993)

7. Evavold, B. D.. Sloan-Lancaster, J. \& Allen, P. M. Proc natn. Acad. Sci. U.S.A. (in the press) 